\title{
新型含取代哌嗪的咖啡因衍生物的合成、结构表征及生物活性研究
}

\author{
张舒旳刘航汪蕾李正名王宝雷* \\ (南开大学化学学院 元素有机化学国家重点实验室 天津 300071)
}

\begin{abstract}
摘要 基于在咖啡因 8-位引入哌嗪活性基团的策略，以 8 -氯茶碱和取代哌嗪为原料，利用 $N$-甲基化、亲核取代、(保护) 脱保护等多步反应，合成了 16 个新型含取代哌嗪的咖啡因衍生物 $\mathbf{I a} \sim \mathbf{I p}$, 通过熔点、 ${ }^{1} \mathrm{H}$ NMR、 ${ }^{13} \mathrm{C}$ NMR 和 HRMS 对 新化合物进行了结构确认和表征，获得了 8-(4-(3-溴-1-(3-氯吡啶-2-基)-1H-吡唑-5-甲酰基)哌嗪-1-基)-1,3,7-三甲基-3,7二氢-1H-嘌呤-2,6-二酮(Ip)的单晶结构. 生物活性测试结果表明, 目标化合物大多对小菜蛾(Plutella xylostella L.)和东方 粘虫(Mythimna separata Walker)表现出显著的杀虫活性, 尤其针对小菜蛾, 部分化合物活性优于咖啡因, 其中 8-(4-(4氟苯基)哌嗪-1-基)-1,3,7-三甲基-3,7-二氢-1H-嘌呤-2,6-二酮(Ie)和 1,3,7-三甲基-8-(4-(4-硝基苯甲酰基)哌嗪-1-基)-3,7-二 氢- $1 H$-嘌呤-2,6-二酮(In)在 $100 \mathrm{mg} \cdot \mathrm{L}^{-1}$ 分别具有 $60 \%$ 和 $53 \%$ 的致死率. 此外, 部分化合物在 $50 \mathrm{mg} \cdot \mathrm{L}^{-1}$ 测试浓度下对苹 果轮纹病菌(Physalospora piricola)、小麦纹枯病菌(Rhizoctonia cerealis)和油菜菌核病菌(Sclerotinia sclerotiorum)等具有 $50 \%$ 以上的抑制活性. 研究结果可为基于黄嘌呤类天然产物结构的新型农药分子设计提供重要参考.
\end{abstract}

关键词 黄嘌呤; 咖啡因; 哌嗪; 合成; 杀虫活性; 抑菌活性

\section{Studies on the Synthesis, Structural Characterization and Biological Activities of Novel Caffeine Derivatives Containing Substituted- Piperazine Moiety}

\author{
Zhang, Shuyun Liu, Hang Wang, Lei Li, Zhengming Wang, Baolei* \\ (State Key Laboratory of Elemento-organic Chemistry, College of Chemistry, Nankai University, Tianjin 300071)
}

\begin{abstract}
Based on a strategy of introducing the "piperazine" bioactive group into 8-position of caffeine, sixteen novel caffeine derivatives containing substituted-piperazine moiety $(\mathbf{I a} \sim \mathbf{I p})$ have been synthesized using 8-chlorotheophylline and substituted-piperazine as materials, via multi step reactions of $N$-methylation, nucleophilic substitution, (protection)deprotection, etc. The structures of the title compounds were confirmed and characterized by melting point, ${ }^{1} \mathrm{H} N M R,{ }^{13} \mathrm{C}$ NMR, and HRMS. The single-crystal structure of 8-(4-(3-bromo-1-(3-chloropyridin-2-yl)-1H-pyrazole-5-carbonyl)piperazin1-yl)-1,3,7-trimethyl-3,7-dihydro-1H-purine-2,6-dione (Ip) was obtained. The bioassay results showed that most of the title compounds possess good insecticidal activities against Plutella xylostella L. and Mythimna separata Walker. In particular, some of the compounds exhibited better larvicidal effect towards Plutella xylostella L. than that of control caffeine, among which 8-(4-(4-fluorophenyl)piperazin-1-yl)-1,3,7-trimethyl-3,7-dihydro-1 $H$-purine-2,6-dione (Ie) and 1,3,7-trimethyl-8-(4-(4nitrobenzoyl)piperazin-1-yl)-3,7-dihydro-1 $H$-purine-2,6-dione (In) at a test concentration of $100 \mathrm{mg} \cdot \mathrm{L}^{-1}$ held lethality rate of $60 \%$ and $53 \%$, respectively. In addition, partial compounds at $50 \mathrm{mg} \cdot \mathrm{L}^{-1}$ concentration possessed $>50 \%$ fungicidal activities against Physalospora piricola, Rhizoctonia cerealis, Sclerotinia sclerotiorum, etc. The research results in this paper provide useful reference for further design of novel agrochemicals based on the natural product structure of xanthine.

Keywords xanthine; caffeine; piperazine; synthesis; insecticidal activity; fungicidal activity
\end{abstract}

众所周知，农药、化肥等农用化学品对于农作物的 保产增收具有不可替代的作用 ${ }^{[1]}$. 近年来, 随着人们对 生态环境保护意识的提高, 以及一些传统农药因农业病
虫草害逐渐产生抗药性而遭到禁用，不断开发高效、低 毒、对生态环境友好的新型农药活性分子成为了当前农 药学的研究热点之一 ${ }^{[2]}$. 黄嘌呤类化合物作为一类天然

\footnotetext{
* Corresponding author. E-mail: nkwbl@nankai.edu.cn

Received May 11, 2021; revised June 23, 2021; published online July 12, 2021.

Dedicated to the 100th anniversary of Chemistry at Nankai University.

Project supported by the National Natural Science Foundation of China (Nos. 21772103, 22077070).

国家自然科学基金(Nos. 21772103, 22077070)资助项目。
} 
产物而广泛存在于自然界, 茶、咖啡、可可、巧克力等 植物中都含有多种形式的黄嘌呤生物碱, 例如咖啡因、 茶碱、可可碱等 ${ }^{[3]}$. 这类化合物和人们的饮食生活密切 相关, 对人体产生的毒性很低. 另外, 其用于治疗疾病 的药用价值也广为人知, 各种天然和人工合成的黄嘌呤 及其衍生物可用于治疗呼吸疾病 ${ }^{[4]}$ 、神经退行性疾病 ${ }^{[5]}$ 、 肾病 ${ }^{[6]}$ 、高血压和心血管疾病 ${ }^{[7]}$. 其在农药领域的研究也 有报道, 但主要在于对咖啡因等天然甲基黄嘌呤生物碱 的研究方面, 例如: 在较低浓度下, 咖啡因能够杀死甲 虫幼虫 ${ }^{[8]}$; 天然和合成的简单甲基黄嘌呤衍生物能够抑 制昆虫摄食; 甲基黄嘌呤是已知可激活昆虫腺苷酸环化 酶的有效增效剂 [9], 同时也是昆虫鱼尼丁受体的激活 剂 ${ }^{[10]}$. 虽然这些证据表明黄嘌呤类化合物可能具有杀 虫等广泛农药活性, 然而关于此方面的研究报道不多 见. 因此基于黄嘌呤骨架结构进行新型农药活性分子设 计有着较大的研究空间和开发潜力.

哌嗪类化合物因其独特的生物活性在医药领域有 着广泛的应用. 同时, 哌嗪环具有低毒、易形成多个氢 键或离子键的特点, 以及调节药物脂水分配系数和酸碱 平衡常数等功能, 常作为药物设计的药效团被引入到不 同类型的结构中以改变母体结构的性质 ${ }^{[11-12]}$; 其在农药 领域的报道主要体现在杀菌活性方面, 例如, 杀菌剂嗪 胺灵(Triforine) ${ }^{[13]}$ 结构中即含有哌嗪结构. 我们在近年 的研究中发现, 一些含哌嗪基团的杂环衍生物可对多种 常见农作物致病真菌表现出具有优异的杀菌活性 ${ }^{[14-18]}$,

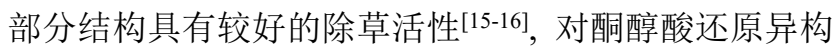
酶(KARI) 表现出良好的抑制活性 ${ }^{[16]}$, 一些化合物还具 有较好的杀虫活性 ${ }^{[19]}$, 这在现有杀虫剂结构中不多见. 综合上述几方面, 本文在甲基黄嘌呤衍生物咖啡因的结 构骨架基础上, 通过活性基团拼接原理, 对其 8-位进行 修饰, 引入取代哌嗪片段, 合成了一系列新型含取代哌 嗪的咖啡因衍生物, 并对新化合物进行结构表征、杀虫 和杀菌农药活性测试, 讨论构效关系. 新化合物结构的 总体设计思路是利用不同取代哌嗪环在生物体内可能 形成的氢键/离子键作用和相关性质调节功能, 将其引 入到咖啡因母体结构骨架中以期提高生物活性.

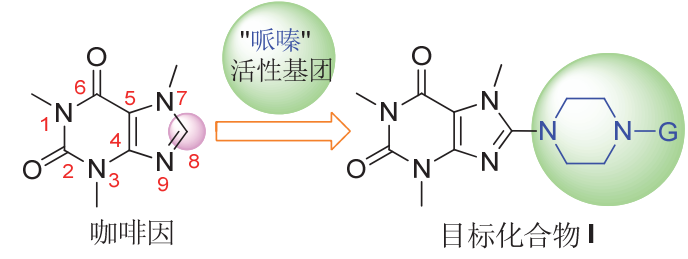

图 1 目标化合物 $\mathbf{I}$ 的设计

Figure 1 Design of the title compounds I

\section{1 结果与讨论}

\section{1 化合物的合成}

如 Scheme 1 所示路线, 以中间体 1-Boc-哌嗪 1 和 4/5-取代-2-氯嘧啶 $2 \mathbf{a} \sim 2 \mathbf{c}$ 为原料, 参考文献[20]方法, 先通过亲核取代反应制得中间体 $\mathbf{3 a} \sim \mathbf{3} \mathbf{c}$, 再与三氟乙 酸在二氯甲烷(DCM)中反应，脱去 Boc 保护基得到含嘧 啶基的哌嗪中间体 $\mathbf{4 a} \sim \mathbf{4 c}$. 按照 Scheme 2 所示路线, 8氯茶碱 5 和碘甲烷以无水碳酸钾作碱, $N, N$-二甲基甲酰 胺 $(\mathrm{DMF})$ 为溶剂、室温下反应得到关键中间体 8-氯咖啡 因 6. 中间体 6 与不同取代的哌嗪中间体在 $\mathrm{K}_{2} \mathrm{CO}_{3}$ 作碱、 $\mathrm{DMF}$ 作溶剂、 $80{ }^{\circ} \mathrm{C}$ 条件下反应 $24 \mathrm{~h}$, 可顺利得到目标 化合物 Ia $\sim \mathbf{I i}$ 和 $\mathbf{I j} \sim \mathbf{I I}$; 中间体 $\mathbf{6}$ 在同样条件下与 1-Boc哌嗪 1 反应得到含 Boc 哌嗪取代的甲基黄嘌呤中间体 7, 再在 $\mathrm{CF}_{3} \mathrm{CO}_{2} \mathrm{H} / \mathrm{DCM}$ 体系中脱去 Boc 保护基得到中 间体 8 . 中间体 8 进而与酰氯发生亲核加成一消除反应, 即可高产率(69\% 90\%) 获得目标产物 Im Ip.

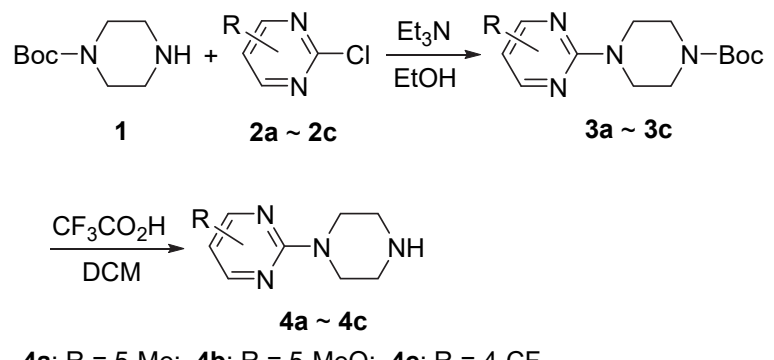

图式 1 中间体 $4 a \sim 4 c$ 的合成路线

Scheme 1 Synthetic route of the intermediates $4 a \sim 4 c$

\section{2 化合物的波谱分析}

目标化合物 $\mathbf{I a} \sim \mathbf{I p}$ 除甲基黄嘌呤骨架结构外，还 含有特殊的取代哌嗪六元环结构, 其 ${ }^{1} \mathrm{H}$ NMR 中典型的 质子共振峰体现在哌嗪环上的 $\mathrm{CH}_{2}$ 大多呈现多重峰, 这 是由于哌嗪环采用类似环己烷的稳定的椅式构象(由下 述单晶结构进一步证明)，六元环上每个亚甲基上的两 个氢处于不同的化学环境中, 其化学位移在 $\delta 4.05$ 3.18. 化合物 Io 结构中含有肉桂酰基, 其 “ $\mathrm{CH}=\mathrm{CH}$ ” 双键碳上两个氢的偶合常数为 $15.4 \mathrm{~Hz}$, 表明该化合物 烯基双键为 $E$ 式构型.

在目标化合物的 ${ }^{13} \mathrm{C} \mathrm{NMR}$ 中, $\delta 151$ 和 155 处分别 为黄嘌呤骨架中两个羰基碳的吸收峰, 与哌嗪环相连的 黄嘌呤的 8 -位碳共振信号出现在 $\delta 161.5 \sim 154.5$ 处. 哌 嗪环中的 4 个亚甲基 $\left(\mathrm{CH}_{2}\right)$ 碳化学位移则在 $\delta 50.8 \sim 44.1$ 处. 此外, 化合物 Ig 的 ${ }^{13} \mathrm{C}$ NMR 中在 $\delta 124.6$ (q, $J=$ $271.5 \mathrm{~Hz}$ )处的四重峰为典型的三氟甲基碳的共振吸收 峰, $\delta 121.4(\mathrm{q}, J=33.0 \mathrm{~Hz})$ 处的四重峰则归属于与三氟 


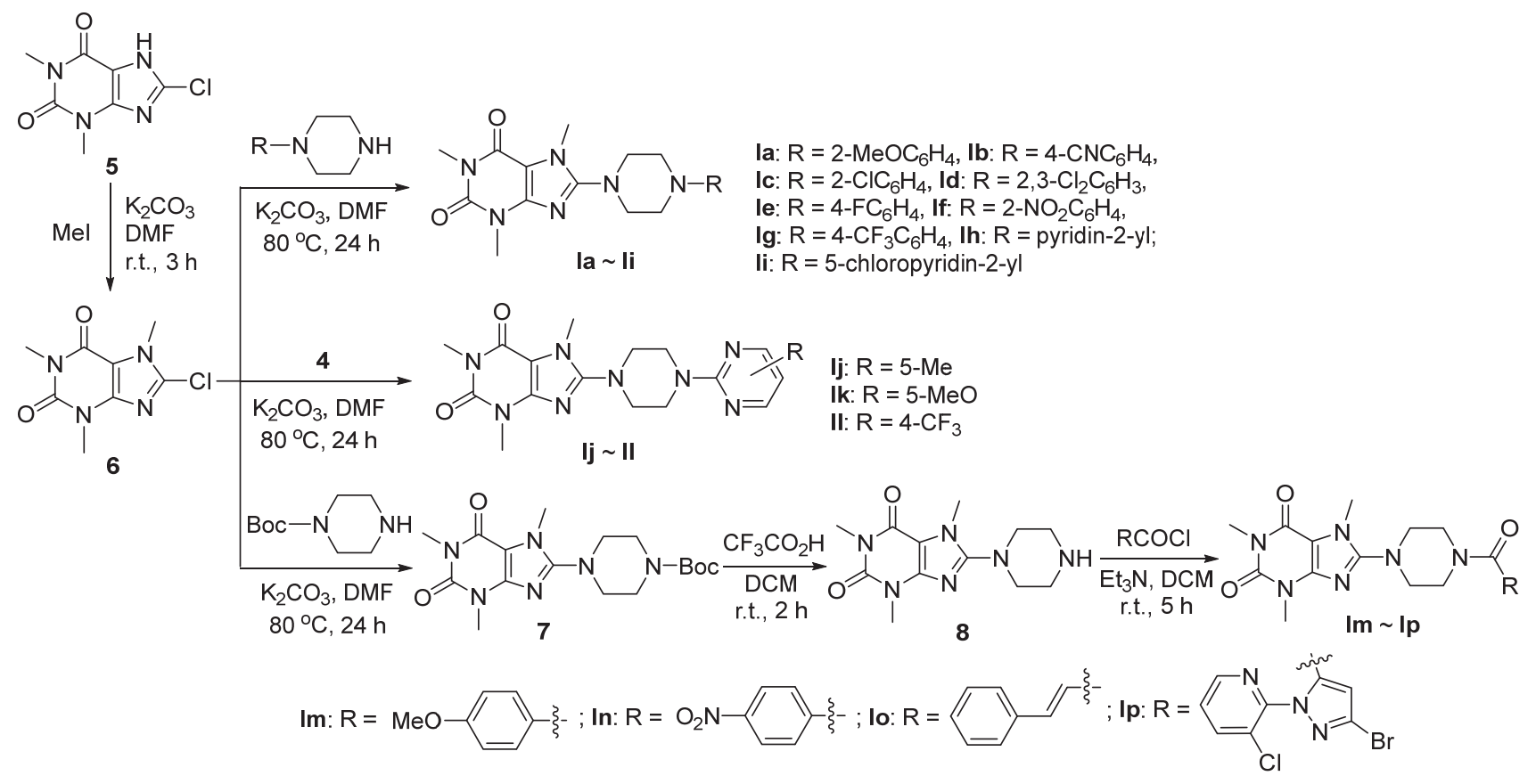

图式 2 目标化合物 $\mathbf{I a} \sim \mathbf{I p}$ 的合成路线

Scheme 2 Synthetic route of the title compounds Ia $\sim$ Ip

甲基直接相连的苯环碳的共振吸收峰, 在 $\delta 126.6$ (q, $J=3.7 \mathrm{~Hz}$ )处, 与三氟甲基相连的苯环上的邻位碳也出 现了裂分现象.

\section{3 晶体结构}

由图 2 的晶体结构(CCDC 号: 2080768)分析表明, 该分子含有甲基黄嘌呤骨架、一个哌嗪环、一个吡唑环 和一个吡啶环. 黄嘌呤骨架的嘧啶二酮六元环平面 $\mathrm{C}(6)-\mathrm{C}(5)-\mathrm{C}(3)-\mathrm{N}(2)-\mathrm{C}(2)-\mathrm{N}(1)$ 与咪唑五元环平面 $\mathrm{C}(3)-\mathrm{C}(5)-\mathrm{N}(4)-\mathrm{C}(4)-\mathrm{N}(3)$ 的二面角为 $3.116^{\circ}$, 表明两个 环几乎共面. 该分子的吡唑环 $\mathrm{C}(14)-\mathrm{C}(15)-\mathrm{C}(16)-\mathrm{N}(7)-$ $\mathrm{N}(8)$ 与吡啶环 $\mathrm{N}(9)-\mathrm{C}(18)-\mathrm{C}(19)-\mathrm{C}(20)-\mathrm{C}(21)-\mathrm{C}(17)$ 的二 面角为 $48.683^{\circ}$, 可能因两个环的邻位均带有较大体积 取代基使之不共平面, 而彼此扭转一定角度对于消除空 间障碍是有利的. 至于哌嗪环中的键角, 例如 $\mathrm{N}(5)-$ $\mathrm{C}(12)-\mathrm{C}(11)$ 为 $111.621^{\circ}, \mathrm{N}(6)-\mathrm{C}(11)-\mathrm{C}(12)$ 为 $111.335^{\circ}, \mathrm{H}(12 \mathrm{~B})-\mathrm{C}(12)-\mathrm{H}(12 \mathrm{~A})$ 为 $107.978^{\circ}$, 以及环 中其它键角则基本接近 $109.5^{\circ}$, 表明该分子的哌嗪环与 环已烷类似, 六元环采用稳定的椅式构象, 而哌嗪环中 两个 $\mathrm{N}$ 原子上所连的取代基吡唑甲酰基和黄嘌呤基团 均处于 $e$ 键.

\section{4 生物活性测试结果及构效关系分析}

表 1 列出了目标化合物 $\mathbf{I a} \sim \mathbf{I p}$ 对东方粘虫和小菜 蛾的生物活性测试结果, 可以看出大多数化合物在 500 和 $250 \mathrm{mg} \cdot \mathrm{L}^{-1}$ 测试浓度下对小菜蛾表现出良好的杀虫 活性, 化合物 Ib、Ie、In 和 Ip 在 $100 \mathrm{mg} \cdot \mathrm{L}^{-1}$ 对小菜蛾

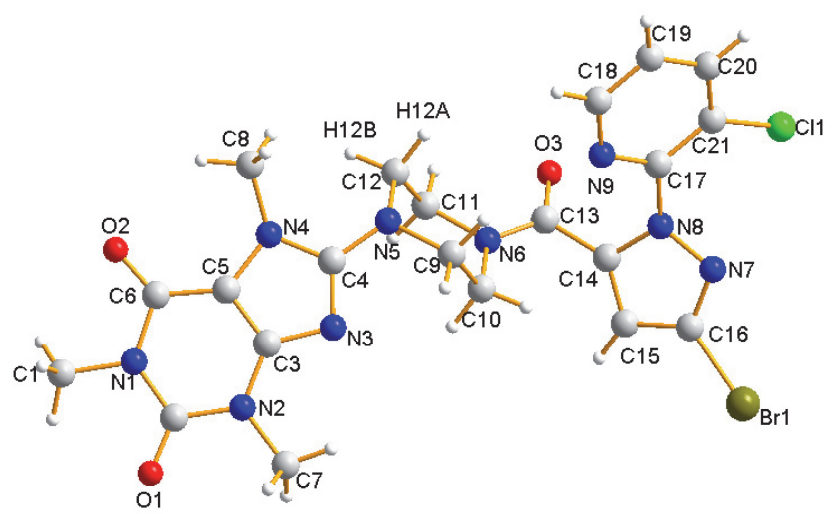

图 2 目标化合物 Ip 的晶体结构

Figure 2 Crystal structure of compound Ip

仍具有 $42 \% \sim 60 \%$ 的致死率，均优于对照化合物咖啡因， 其中活性最好的化合物 Ie 致死率达 $60 \%$. 由构效关系分 析可知, 当与哌嗪直接相连的基团为芳基时, 芳基为取 代苯环的化合物的杀虫活性优于为取代嘧啶和取代吡 啶的化合物, 而化合物苯环上所带给电子取代基 $\mathrm{CH}_{3} \mathrm{O}$ 或吸电子取代基 $\mathrm{CN}$ 和 $\mathrm{F}$ 均能导致较高的活性, 哌嗪环 上取代基相对应化合物的活性顺序为: 4-氟苯基 $>4$-氧 基苯基 $>2$-甲氧基苯基. 当哌嗪上带有酰基取代基时, 对于与羰基相连的芳香基团而言, 其上含有吸电子取代 基的杀虫活性优于含有给电子基，例如为硝基时活性远 高于为甲氧基的活性. 当与羰基相连的基团为取代吡唑 基(Ip)和苯乙烯基(Io)时，化合物在 $100 \mathrm{mg} \cdot \mathrm{L}^{-1}$ 时对小 菜蛾分别具有 $42 \%$ 和 $37 \%$ 的杀虫致死率. 此外, 部分化 
表 1 目标化合物 $\mathbf{I}$ 的生物活性

Table 1 Biological activities of the title compounds I

\begin{tabular}{|c|c|c|c|c|c|c|c|c|c|c|}
\hline \multirow{3}{*}{ Compd. } & \multicolumn{4}{|c|}{ 杀虫活性，致死率\% } & \multicolumn{6}{|c|}{ 离体抑菌活性 $\left(50 \mathrm{mg} \cdot \mathrm{L}^{-1}\right)$, 抑制率\% } \\
\hline & \multirow{2}{*}{$\frac{\text { 东方粘虫 }}{500 \mathrm{mg} \cdot \mathrm{L}^{-1}}$} & \multicolumn{3}{|c|}{ 小菜蛾 } & \multirow{2}{*}{$\begin{array}{l}\text { 番茄早 } \\
\text { 疫病菌 }\end{array}$} & \multirow{2}{*}{$\begin{array}{l}\text { 辣椒疫 } \\
\text { 霉病菌 }\end{array}$} & \multirow{2}{*}{$\begin{array}{l}\text { 油菜菌 } \\
\text { 核病菌 }\end{array}$} & \multirow{2}{*}{$\begin{array}{l}\text { 黄瓜灰 } \\
\text { 霉病菌 }\end{array}$} & \multirow{2}{*}{$\begin{array}{l}\text { 苹果轮 } \\
\text { 纹病菌 }\end{array}$} & \multirow{2}{*}{$\begin{array}{l}\text { 小麦纹 } \\
\text { 枯病菌 }\end{array}$} \\
\hline & & $500 \mathrm{mg} \cdot \mathrm{L}^{-1}$ & $250 \mathrm{mg} \cdot \mathrm{L}^{-1}$ & $100 \mathrm{mg} \cdot \mathrm{L}^{-1}$ & & & & & & \\
\hline Ia & 5 & 100 & 70 & 28 & 29.4 & 13.9 & 30.4 & 18.2 & 71.1 & 55.4 \\
\hline Ib & 50 & 100 & 80 & 47 & 5.9 & 5.6 & 17.9 & 4.5 & 47.4 & 27.7 \\
\hline Ic & 45 & 79 & 43 & NT & 17.6 & 5.6 & 7.1 & 13.6 & 63.2 & 29.2 \\
\hline Id & 60 & 70 & 32 & NT & 29.4 & 13.9 & 50.0 & 27.3 & 71.1 & 40.0 \\
\hline Ie & 0 & 100 & 92 & 60 & 5.9 & 13.9 & 3.6 & 27.3 & 52.6 & 56.9 \\
\hline If & 20 & 90 & 63 & NT & 17.6 & 11.1 & 17.9 & 4.5 & 5.3 & 32.3 \\
\hline Ig & 0 & 88 & 55 & NT & 11.8 & 13.9 & 8.9 & 13.6 & 44.7 & 30.8 \\
\hline Ih & 30 & 43 & NT & NT & 23.5 & 8.3 & 7.1 & 27.3 & 31.6 & 24.6 \\
\hline $\mathbf{I i}$ & 20 & 65 & NT & NT & 17.6 & 13.9 & 26.8 & 13.6 & 57.9 & 41.5 \\
\hline $\mathbf{I j}$ & 15 & 53 & NT & NT & 35.3 & 22.2 & 26.8 & 18.2 & 31.6 & 29.2 \\
\hline Ik & 60 & 18 & NT & NT & 5.9 & 8.3 & 25.0 & 27.3 & 52.6 & 27.7 \\
\hline Il & 5 & 58 & NT & NT & 5.9 & 19.4 & 7.1 & 27.3 & 13.2 & 53.8 \\
\hline Im & 20 & 30 & NT & NT & 11.8 & 13.9 & 32.1 & 18.2 & 0 & 52.3 \\
\hline In & 0 & 100 & 87 & 53 & 17.6 & 11.1 & 8.9 & 4.5 & 0 & 29.2 \\
\hline Io & 10 & 100 & 71 & 37 & 23.5 & 5.6 & 7.1 & 13.6 & 23.7 & 21.5 \\
\hline Ip & 50 & 100 & 77 & 42 & 17.6 & 8.3 & 14.3 & 22.7 & 57.9 & 27.7 \\
\hline $\mathrm{C}-1^{a}$ & 65 & 88 & 30 & NT & 5.3 & 8.8 & 8.3 & 9.4 & 37.3 & 13.2 \\
\hline$C-2^{b}$ & $\mathrm{NT}^{d}$ & NT & NT & NT & 100.0 & 82.4 & 100.0 & 50.0 & 98.5 & 75.0 \\
\hline $\mathrm{C}-3^{c}$ & NT & NT & NT & NT & 26.3 & 73.5 & 91.7 & 81.3 & 91.0 & 81.6 \\
\hline
\end{tabular}

${ }^{a} \mathrm{C}-1=$ caffeine. ${ }^{b} \mathrm{C}-2=$ azoxystrobin. ${ }^{c} \mathrm{C}-3=$ chlorothalonil. ${ }^{d} \mathrm{NT}=$ not test.

合物在 $500 \mathrm{mg} \cdot \mathrm{L}^{-1}$ 时对东方粘虫也具有显著的杀虫活 性，其中化合物 Ib、Id、Ik 和 Ip 的致死率达 50\% 60\%, 与咖啡因活性接近.

表 1 还列出了目标化合物 $\mathbf{I a} \sim \mathbf{I p}$ 对 6 种供试植物 病菌的生物活性测试结果，表明大多数化合物在 50 $\mathrm{mg} \cdot \mathrm{L}^{-1}$ 测试浓度下对苹果轮纹病菌表现出较好的抑菌 活性, 多数化合物对苹果轮纹病菌的抑制率超过 $50 \%$, 尤其化合物 Ia、Id 具有 $71.1 \%$ 的抑制率, 活性远超对照 化合物咖啡因, 但低于对照药嘧菌酯和百菌清. 由构效 关系分析可知, 当与哌嗪直接相连的基团为杂芳基时, 相较于酰基, 对苹果轮纹病菌的抑制活性较好, 且半数 化合物活性超过 50\%; 当直接相连基团为酰基时, 仅有 化合物 Ip 抑菌活性超过 50\%; 当杂芳基上的取代基为 氯、氟或甲氧基时对苹果轮纹病菌的抑菌活性优于为其 他基团的活性. 总体来看, 当哌嗪直接相连的基团为杂 芳基时，哌嗪环上取代基相对应化合物的活性顺序为: 2 -甲氧基苯基 $\approx 2,3$-二氯苯基 $>2$-氯苯基 $>5$-氯吡啶-2基 $>5$-甲氧基嘧啶- 2 -基 $\approx 4$-氟苯基 $>4$-氰基苯基 $>4$-三 氟甲基苯基 $>5$-甲基嘧啶-2-基 $>4$-三氟甲基嘧啶-2基 $>2$-硝基苯基. 部分化合物对小麦纹枯病菌有一定的 抑制活性，其中化合物 Ia、Ie、Il 和 Im 的抑菌活性超 过 $50 \%$. 另外, 化合物 Id 对油菜菌核病菌也可表现出 $50 \%$ 的抑菌活性.

\section{2 结论}

以 8 -氯茶碱和取代哌嗪为原料，通过 $N$-甲基化、亲 核取代、(保护)脱保护等多步反应, 合成了 16 个未见文 献报道的新型含取代哌嗪的咖啡因衍生物 $\mathbf{I a} \sim \mathbf{I p}$, 通过 ${ }^{1} \mathrm{H}$ NMR、 ${ }^{13} \mathrm{C} \mathrm{NMR}$ 和 HRMS 确证了新化合物的结构, 获得了化合物 Ip 的单晶结构. 生物活性测试结果表明, 大部分目标化合物对小菜蛾和东方粘虫表现出显著的 杀虫活性，尤其针对小菜蛾，部分化合物活性优于对照 化合物咖啡因, 其中化合物 $\mathbf{I e}$ 和 In 在 $100 \mathrm{mg} \cdot \mathrm{L}^{-1}$ 分别 具有 $60 \%$ 和 $53 \%$ 的致死率. 此外, 部分化合物对苹果轮 纹病菌、小麦纹枯病菌和油菜菌核病菌等具有 $50 \%$ 以上 的抑制活性. 本文研究结果对基于甲基黄嘌呤结构的新 型农药分子设计具有一定的参考价值.

\section{3 实验部分}

\section{1 仪器与试剂}

X-4 型数字显示显微熔点仪(温度计未校正); Bruker Avance 400 型核磁共振仪(以 TMS 为内标); Bruker solariX XR 型傅立叶变换高分辨质谱仪; 007HF XtaLAB P200 (Mo 靶)型单晶衍射仪. 所用试剂均为国产分析纯, 使用前未经处理. 


\section{2 实验方法}

\subsection{1 中间体取代嘧啶基哌嗪 $\mathbf{4 a} \sim \mathbf{4} \mathbf{c}$ 的合成}

中间体取代嘧啶基哌嗪 $\mathbf{4 a} \sim \mathbf{4}$ 按照文献[20]合成.

5-甲基-2-(哌嗪-1-基)嘧啶(4a)：黄色油状物, 产率 $70.4 \%$. 核磁共振氢谱数据与文献[20]一致.

5-甲氧基-2-(哌嗪-1-基)嘧啶(4b)：黄色油状物, 产 率 $63.8 \%$. 核磁共振氢谱数据与文献[20]一致.

4-三氟甲基-2-(哌嗪-1-基)嘧啶(4c)：黄色粘稠物， 产率 19.6\%. ${ }^{1} \mathrm{H}$ NMR $\left(400 \mathrm{MHz}, \mathrm{CDCl}_{3}\right) \delta: 8.55 \sim 8.45$ (m, $1 \mathrm{H}$, pyrimidine- $\mathrm{H}), 6.78 \sim 6.73(\mathrm{~m}, 1 \mathrm{H}$, pyrimidine- $\mathrm{H})$, $3.95 \sim 3.80\left(\mathrm{~m}, 4 \mathrm{H}\right.$, piperazine- $\left.\mathrm{CH}_{2}\right), 3.10 \sim 2.85(\mathrm{~m}, 4 \mathrm{H}$, piperazine- $\left.\mathrm{CH}_{2}\right), 2.14(\mathrm{~s}, 1 \mathrm{H}, \mathrm{NH})$.

3.2.2 中间体 1,3,7-三甲基-3,7-二氢-1 $H$-嘌呤-2,6-二 酮(6)的合成

向 $250 \mathrm{~mL}$ 圆底烧瓶中加入 8-氯茶碱(5) (10.7 g, 50 $\mathrm{mmol}) 、$ 无水碳酸钾 $(13.8 \mathrm{~g}, 100 \mathrm{mmol})$ 和 $150 \mathrm{~mL} N, N$-二 甲基甲酰胺(DMF), 磁力搅拌使其混合均匀, 再加入碘 甲烷(14.1 g, $100 \mathrm{mmol})$, 室温搅拌 $1 \mathrm{~h}$. 过滤, 滤饼依次 用水和乙醇洗涤, 干燥得 $21.1 \mathrm{~g}$ 中间体 $\mathbf{6}$, 产率 $92.4 \%$. 白色固体, m.p. $186 \sim 188{ }^{\circ} \mathrm{C}$ (lit. ${ }^{[21]}$ m.p. $188{ }^{\circ} \mathrm{C}$ ); ${ }^{1} \mathrm{H}$ NMR (400 MHz, $\left.\mathrm{CDCl}_{3}\right) \delta: 3.95$ (s, 3H, $\left.\mathrm{NCH}_{3}\right), 3.54$ (s, $\left.3 \mathrm{H}, \mathrm{NCH}_{3}\right), 3.39\left(\mathrm{~s}, 3 \mathrm{H}, \mathrm{NCH}_{3}\right)$.

3.2.3中间体 4-((1,3,7-三甲基-2,6-二氧杂- $2,3,6,7$-四 氢-1 $H$-嘌呤-8-基))哌嗪-1-甲酸叔丁酯(7)的合成

向 $100 \mathrm{~mL}$ 圆底烧瓶中加入中间体 $6(2.28 \mathrm{~g}, 10$ $\mathrm{mmol}) 、$ 无水碳酸钾 $(2.76 \mathrm{~g}, 20 \mathrm{mmol}) 、 1-B o c-$ 哌嗪(1.86 g, $10 \mathrm{mmol}$ )和 $15 \mathrm{~mL} \mathrm{DMF}$, 将混合物于 $80{ }^{\circ} \mathrm{C}$ 下磁力搅拌 反应 $24 \mathrm{~h}$ [薄层色谱(TLC)监测]. 待反应完全后, 冷却, 向体系中倒入 $100 \mathrm{~mL}$ 水中, 混合物用二氯甲烷萃取(30 $\mathrm{mL} \times 3)$, 合并有机相, 用无水硫酸钠干燥, 减压浓缩除 去约 $2 / 3$ 体积的溶剂, 即有固体析出, 抽滤, 用水洗涤, 干燥, 得中间体 7, 产率 72.3\%. 白色固体, m.p. 200 $201{ }^{\circ} \mathrm{C} ;{ }^{1} \mathrm{H}$ NMR $\left(400 \mathrm{MHz}, \mathrm{CDCl}_{3}\right) \delta: 3.79(\mathrm{~s}, 3 \mathrm{H}$, $\left.\mathrm{NCH}_{3}\right), 3.66 \sim 3.58\left(\mathrm{~m}, 4 \mathrm{H}\right.$, piperazine- $\left.\mathrm{CH}_{2}\right), 3.55(\mathrm{~s}, 3 \mathrm{H}$, $\left.\mathrm{NCH}_{3}\right), 3.41\left(\mathrm{~s}, 3 \mathrm{H}, \mathrm{NCH}_{3}\right), 3.28 \sim 3.21(\mathrm{~m}, 4 \mathrm{H}$, piperazine- $\left.\mathrm{CH}_{2}\right), 1.50\left(\mathrm{~s}, 9 \mathrm{H}, 3 \times\right.$ Boc- $\left.\mathrm{CH}_{3}\right) ;{ }^{13} \mathrm{C}$ NMR $(101$ $\left.\mathrm{MHz}, \mathrm{CDCl}_{3}\right) \delta: 155.9,155.0,154.6,151.71,105.5,49.6$, 32.4, 29.7, 28.4, 27.8; HRMS (Maldi) calcd for $\mathrm{C}_{17} \mathrm{H}_{27}-$ $\mathrm{N}_{6} \mathrm{O}_{4}[\mathrm{M}+\mathrm{H}]^{+}$379.2089, found 379.2088.

3.2.4 中间体 1,3,7-三甲基-8-(哌嗪-1-基)-3,7-二氢$1 H$-嘌呤-2,6-二酮 $(8)$ 的合成

向 $100 \mathrm{~mL}$ 圆底烧瓶中加入中间体 7 (3.78 g, 10 $\mathrm{mmol}$ )和 $20 \mathrm{~mL}$ 二氯甲烷, 磁力搅拌使固体溶解, 再加 入 $5 \mathrm{~mL}$ 三氟乙酸, 室温搅拌 $2 \mathrm{~h}$, 将混合物转移到 250 $\mathrm{mL}$ 烧杯中. 向体系中加入 $50 \mathrm{~mL}$ 水和 $50 \mathrm{~mL}$ 乙酸乙酯,
将混合物转入分液漏斗, 充分振荡, 分离水相. 用碳酸 钾调节水相 $\mathrm{pH}$ 为 10 后，再用二氯甲烷萃取 $(30 \mathrm{~mL} \times 3)$, 合并有机相, 用无水硫酸钠干燥, 减压脱溶, 得中间体 8, 产率 83.7\%. 白色固体, m.p. $180 \sim 181{ }^{\circ} \mathrm{C}$ (lit. ${ }^{[22]}$ $\left.179{ }^{\circ} \mathrm{C}\right) ;{ }^{1} \mathrm{H}$ NMR $\left(400 \mathrm{MHz}, \mathrm{CDCl}_{3}\right) \delta: 3.75(\mathrm{~s}, 3 \mathrm{H}$, $\left.\mathrm{NCH}_{3}\right), 3.53\left(\mathrm{~s}, 3 \mathrm{H}, \mathrm{NCH}_{3}\right), 3.39\left(\mathrm{~s}, 3 \mathrm{H}, \mathrm{NCH}_{3}\right), 3.27 \sim$ $3.21\left(\mathrm{~m}, 4 \mathrm{H}\right.$, piperazine- $\left.\mathrm{CH}_{2}\right), 3.09 \sim 3.02(\mathrm{~m}, 4 \mathrm{H}$, piperazine- $\left.\mathrm{CH}_{2}\right), 1.72$ (s, 1H, piperazine-NH); ${ }^{13} \mathrm{C}$ NMR (101 $\left.\mathrm{MHz}, \mathrm{CDCl}_{3}\right) \delta: 156.6,154.9,151.8,147.5,105.4,50.9$, 45.6, 32.6, 29.7, 27.7.

\section{2 .5 目标化合物 $\mathbf{I a} \sim \mathbf{I I}$ 的合成}

向 $25 \mathrm{~mL}$ 圆底烧瓶中加入中间体 $6(1.1 \mathrm{mmol}) 、$ 无 水碳酸钾 $(2 \mathrm{mmol}) 、 1$-取代哌嗪或中间体 $4(1 \mathrm{mmol})$ 和 $15 \mathrm{~mL} \mathrm{DMF}$, 将混合物于 $80{ }^{\circ} \mathrm{C}$ 下磁力搅拌反应 $24 \mathrm{~h}$ (TLC 监测). 待反应完全后, 冷却, 将混合物转移到 250 $\mathrm{mL}$ 烧杯中, 向体系中加入 $150 \mathrm{~mL}$ 水, 用二氯甲烷萃取 $(50 \mathrm{~mL} \times 3)$. 合并有机相, 用无水硫酸钠干燥, 减压浓 缩除去约 $2 / 3$ 体积的溶剂即有固体析出，抽滤，用水洗 涤, 干燥, 得到目标化合物 $\mathbf{I a} \sim \mathbf{I I}$.

8-(4-(2-甲氧基苯基)哌嗪-1-基)-1,3,7-三甲基-3,7-二 氢- $1 H$-嘌呤-2,6-二酮 (Ia): 淡黄色固体，产率 $42.1 \%$. m.p. $165{ }^{\circ} \mathrm{C} ;{ }^{1} \mathrm{H}$ NMR $\left(400 \mathrm{MHz}, \mathrm{CDCl}_{3}\right) \delta: 7.08 \sim 7.03$ $(\mathrm{m}, 1 \mathrm{H}, \mathrm{PhH}), 6.99 \sim 6.94(\mathrm{~m}, 2 \mathrm{H}, \mathrm{PhH}), 6.91(\mathrm{~d}, J=7.8$ $\mathrm{Hz}, 1 \mathrm{H}, \mathrm{PhH}), 3.89$ (s, $\left.3 \mathrm{H}, \mathrm{OCH}_{3}\right), 3.79\left(\mathrm{~s}, 3 \mathrm{H}, \mathrm{NCH}_{3}\right)$, $3.55\left(\mathrm{~s}, 3 \mathrm{H}, \mathrm{NCH}_{3}\right), 3.50 \sim 3.47\left(\mathrm{~m}, 4 \mathrm{H}\right.$, piperazine- $\left.\mathrm{CH}_{2}\right)$, $3.40\left(\mathrm{~s}, 3 \mathrm{H}, \mathrm{NCH}_{3}\right), 3.25 \sim 3.21$ (m, $4 \mathrm{H}$, piperazine- $\mathrm{CH}_{2}$ ); ${ }^{13} \mathrm{C}$ NMR (101 MHz, $\left.\mathrm{CDCl}_{3}\right) \delta: 156.4,154.9,152.2,151.7$, $147.5,140.7,123.4,121.0,118.36,111.4,105.4,55.4$, 50.1, 49.9, 32.6, 29.7, 27.7; HRMS (Maldi) calcd for $\mathrm{C}_{19} \mathrm{H}_{25} \mathrm{~N}_{6} \mathrm{O}_{3}[\mathrm{M}+\mathrm{H}]^{+}$385.1983, found 385.1980 .

4-(4-(1,3,7-三甲基-2,6-二氧代-2,3,6,7-四氢- $1 H$-嘌 呤-8-基)哌嗪-1-基)芐腈(Ib)：黄色固体，产率 45.9\%. m.p. $248{ }^{\circ} \mathrm{C}$; ${ }^{1} \mathrm{H}$ NMR $\left(400 \mathrm{MHz}, \mathrm{CDCl}_{3}\right) \delta$ : $7.49 \sim 7.47$ (m, 2H, PhH), 6.87 6.84 (m, 2H, PhH), $3.74(\mathrm{~s}, 3 \mathrm{H}$, $\mathrm{NCH}_{3}$ ), 3.47 (s, $3 \mathrm{H}, \mathrm{NCH}_{3}$ ), 3.42 (dd, $J=6.8,3.2 \mathrm{~Hz}, 4 \mathrm{H}$, piperazine- $\left.\mathrm{CH}_{2}\right), 3.36$ (dd, $J=6.8,3.2 \mathrm{~Hz}, 4 \mathrm{H}$, piperazine$\left.\mathrm{CH}_{2}\right), 3.33$ (s, $\left.3 \mathrm{H}, \mathrm{NCH}_{3}\right) ;{ }^{13} \mathrm{C} \mathrm{NMR}\left(101 \mathrm{MHz}, \mathrm{CDCl}_{3}\right) \delta$ : $154.5,154.0,152.1,150.6,146.2,132.5,118.7,113.6$, 104.5, 100.3, 48.3, 45.9, 31.4, 28.7, 26.8; HRMS (Maldi) calcd for $\mathrm{C}_{19} \mathrm{H}_{22} \mathrm{~N}_{7} \mathrm{O}_{2}[\mathrm{M}+\mathrm{H}]^{+} 380.1829$, found 380.1825 .

8-(4-(2-氯苯基)哌嗪-1-基)-1,3,7-三甲基-3,7-二氢$1 H$-嘌呤-2,6-二酮 (Ic): 白色固体，产率 $68.9 \%$. m.p. $229 \sim 230{ }^{\circ} \mathrm{C} ;{ }^{1} \mathrm{H}$ NMR $\left(400 \mathrm{MHz}, \mathrm{CDCl}_{3}\right) \delta: 7.40(\mathrm{dd}$, $J=7.9,1.5 \mathrm{~Hz}, 1 \mathrm{H}, \mathrm{PhH}), 7.25(\mathrm{~d}, J=7.7,1 \mathrm{H}, \mathrm{PhH}), 7.08$ 
(d, $J=8.0,1 \mathrm{H}, \mathrm{PhH}), 7.02$ (t, $J=7.7 \mathrm{~Hz}, 1 \mathrm{H}, \mathrm{PhH}), 3.80$ (s, $\left.3 \mathrm{H}, \mathrm{NCH}_{3}\right), 3.55\left(\mathrm{~s}, 3 \mathrm{H}, \mathrm{NCH}_{3}\right), 3.50 \sim 3.45(\mathrm{~m}, 4 \mathrm{H}$, piperazine- $\left.\mathrm{CH}_{2}\right), 3.40\left(\mathrm{~s}, 3 \mathrm{H}, \mathrm{NCH}_{3}\right), 3.25 \sim 3.18(\mathrm{~m}, 4 \mathrm{H}$, piperazine- $\left.\mathrm{CH}_{2}\right) ;{ }^{13} \mathrm{C}$ NMR $\left(101 \mathrm{MHz}, \mathrm{CDCl}_{3}\right) \delta: 155.2$, $154.0,150.7,147.7,146.4,129.7,127.9,126.6,123.2$, 119.4, 104.4, 49.7, 48.9, 31.5, 28.7, 26.7; HRMS (Maldi) calcd for $\mathrm{C}_{18} \mathrm{H}_{22} \mathrm{ClN}_{6} \mathrm{O}_{2}[\mathrm{M}+\mathrm{H}]^{+}$389.1487, found 389.1486.

8-(4-(2,3-二氯苯基)哌嗪-1-基)-1,3,7-三甲基-3,7-二 氢-1 $H$-嘌呤-2,6-二酮(Id): 白色固体, 产率 67.4\%. m.p. $265 \sim 267{ }^{\circ} \mathrm{C} ;{ }^{1} \mathrm{H}$ NMR $\left(400 \mathrm{MHz}, \mathrm{CDCl}_{3}\right) \delta: 7.25 \sim 7.17$ (m, 2H, PhH), 7.01 (dd, J=7.5, 2.0 Hz, 1H, PhH), 3.81 (s, $\left.3 \mathrm{H}, \mathrm{NCH}_{3}\right), 3.56\left(\mathrm{~s}, 3 \mathrm{H}, \mathrm{NCH}_{3}\right), 3.52 \sim 3.46(\mathrm{~m}, 4 \mathrm{H}, \mathrm{pi}-$ perazine- $\left.\mathrm{CH}_{2}\right), 3.41\left(\mathrm{~s}, 3 \mathrm{H}, \mathrm{NCH}_{3}\right), 3.26 \sim 3.19(\mathrm{~m}, 4 \mathrm{H}$, piperazine- $\left.\mathrm{CH}_{2}\right) ;{ }^{13} \mathrm{C}$ NMR $\left(101 \mathrm{MHz}, \mathrm{CDCl}_{3}\right) \delta: 156.2$, $155.0,151.8,150.7,147.4,134.2,127.8,127.5,125.2$, 118.7, 105.5, 50.8, 49.9, 32.5, 29.7, 27.8; HRMS (Maldi) calcd for $\mathrm{C}_{18} \mathrm{H}_{21} \mathrm{Cl}_{2} \mathrm{~N}_{6} \mathrm{O}_{2}[\mathrm{M}+\mathrm{H}]^{+}$423.1098, found 423.1103.

8-(4-(4-氟苯基)哌嗪-1-基)-1,3,7-三甲基-3,7-二氢$1 H$-嘌呤-2,6-二酮(Ie): 白色固体, 产率 45.3\%. m.p. $168 \sim 170{ }^{\circ} \mathrm{C} ;{ }^{1} \mathrm{H}$ NMR $\left(400 \mathrm{MHz}, \mathrm{CDCl}_{3}\right) \delta: 7.48(\mathrm{~d}, J=$ $9.0 \mathrm{~Hz}, 2 \mathrm{H}, \mathrm{PhH}), 6.86$ (d, J=9.0 Hz, 2H, PhH), 3.74 (s, $\left.3 \mathrm{H}, \mathrm{NCH}_{3}\right), 3.47$ (s, $\left.3 \mathrm{H}, \mathrm{NCH}_{3}\right), 3.42(\mathrm{dd}, J=6.8,3.3 \mathrm{~Hz}$, $4 \mathrm{H}$, piperazine- $\left.\mathrm{CH}_{2}\right), 3.36(\mathrm{dd}, J=6.8,3.3 \mathrm{~Hz}, 4 \mathrm{H}$, piperazine- $\left.\mathrm{CH}_{2}\right), 3.33\left(\mathrm{~s}, 3 \mathrm{H}, \mathrm{NCH}_{3}\right) ;{ }^{13} \mathrm{C}$ NMR $(101 \mathrm{MHz}$, $\left.\mathrm{CDCl}_{3}\right) \delta: 156.0,155.0,151.7,147.6,147.4,118.3$ (d, $J=$ $7.7 \mathrm{~Hz}), 115.8,115.6,105.5,49.9,49.7,32.5,29.7,27.8$; HRMS (Maldi) calcd for $\mathrm{C}_{18} \mathrm{H}_{22} \mathrm{FN}_{6} \mathrm{O}_{2} \quad[\mathrm{M}+\mathrm{H}]^{+}$ 373.1783 , found 373.1780 .

1,3,7-三甲基-8-(4-(2-硝基苯基)哌嗪-1-基)-3,7-二 氢-1 $H$-嘌呤-2,6-二酮(If): 橙黄色固体, 产率 $61.6 \%$. m.p. $224 \sim 225{ }^{\circ} \mathrm{C}$; ${ }^{1} \mathrm{H}$ NMR $\left(400 \mathrm{MHz}, \mathrm{CDCl}_{3}\right) \delta$ : $7.81(\mathrm{dd}$, $J=8.1,1.5 \mathrm{~Hz}, 1 \mathrm{H}, \mathrm{PhH}), 7.58 \sim 7.50(\mathrm{~m}, 1 \mathrm{H}, \mathrm{PhH}), 7.22$ (dd, $J=8.2,1.0 \mathrm{~Hz}, 1 \mathrm{H}, \mathrm{PhH}), 7.17 \sim 7.10(\mathrm{~m}, 1 \mathrm{H}, \mathrm{PhH})$, 3.79 (s, $3 \mathrm{H}, \mathrm{NCH}_{3}$ ), 3.54 (s, $3 \mathrm{H}, \mathrm{NCH}_{3}$ ), 3.45 (dd, $J=5.9$, $3.7 \mathrm{~Hz}, 4 \mathrm{H}$, piperazine- $\left.\mathrm{CH}_{2}\right), 3.40\left(\mathrm{~s}, 3 \mathrm{H}, \mathrm{NCH}_{3}\right), 3.23$ (dd, $J=5.9,3.7 \mathrm{~Hz}, 4 \mathrm{H}$, piperazine- $\left.\mathrm{CH}_{2}\right) ;{ }^{13} \mathrm{C}$ NMR $(101 \mathrm{MHz}$, $\left.\mathrm{CDCl}_{3}\right) \delta: 154.7,153.8,150.5,146.1,144.4,142.8,132.3$, 124.6, 121.6, 120.3, 104.2, 50.1, 48.5, 31.3, 28.5, 26.5; HRMS (Maldi) calcd for $\mathrm{C}_{18} \mathrm{H}_{22} \mathrm{~N}_{7} \mathrm{O}_{4}[\mathrm{M}+\mathrm{H}]^{+} 400.1728$, found 400.1728 .

1,3,7-三甲基-8-(4-(4-(三氟甲基)苯基)哌嗪-1基)-3,7-二氢-1 $H$-嘌呤-2,6-二酮(Ig): 白色固体, 产率 49.6\%. m.p. $186{ }^{\circ} \mathrm{C} ;{ }^{1} \mathrm{H}$ NMR (400 MHz, $\mathrm{CDCl}_{3}$ ) $\delta: 7.53$ (d, $J=8.7 \mathrm{~Hz}, 2 \mathrm{H}, \mathrm{PhH}), 6.98(\mathrm{~d}, J=8.7 \mathrm{~Hz}, 2 \mathrm{H}, \mathrm{PhH})$, $3.81\left(\mathrm{~s}, 3 \mathrm{H}, \mathrm{NCH}_{3}\right), 3.54\left(\mathrm{~s}, 3 \mathrm{H}, \mathrm{NCH}_{3}\right), 3.44(\mathrm{~s}, 8 \mathrm{H}, \mathrm{pi}-$ perazine- $\left.\mathrm{CH}_{2}\right), 3.40\left(\mathrm{~s}, 3 \mathrm{H}, \mathrm{NCH}_{3}\right) ;{ }^{13} \mathrm{C}$ NMR $(101 \mathrm{MHz}$, $\left.\mathrm{CDCl}_{3}\right) \delta: 155.8,155.0,153.0,151.7,147.3,126.6$ (q, $J=$ $3.7 \mathrm{~Hz}), 124.6(\mathrm{q}, J=271.5 \mathrm{~Hz}), 121.4(\mathrm{q}, J=33.0 \mathrm{~Hz})$, 114.9, 105.5, 49.5, 47.8, 32.4, 29.7, 27.8; HRMS (Maldi) calcd for $\mathrm{C}_{19} \mathrm{H}_{22} \mathrm{~F}_{3} \mathrm{~N}_{6} \mathrm{O}_{2}[\mathrm{M}+\mathrm{H}]^{+}$423.1751, found 423.1748 .

1,3,7-三甲基-8-(4-(吡啶-2-基)哌嗪-1-基)-3,7-二氢$1 H$-嘌呤-2,6-二酮(Ih): 白色固体，产率 54.7\%. m.p. $223{ }^{\circ} \mathrm{C}$; ${ }^{1} \mathrm{H}$ NMR $\left(400 \mathrm{MHz}, \mathrm{CDCl}_{3}\right) \delta: 8.24 \sim 8.20(\mathrm{~m}$, $1 \mathrm{H}$, pyridine-H), $7.58 \sim 7.50(\mathrm{~m}, 1 \mathrm{H}$, pyridine- $\mathrm{H}), 6.73 \sim$ $6.66(\mathrm{~m}, 2 \mathrm{H}$, pyridine- $\mathrm{H}), 3.81\left(\mathrm{~s}, 3 \mathrm{H}, \mathrm{NCH}_{3}\right), 3.73 \sim 3.68$ (m, $4 \mathrm{H}$, piperazine- $\left.\mathrm{CH}_{2}\right), 3.54$ (s, $\left.3 \mathrm{H}, \mathrm{NCH}_{3}\right), 3.42 \sim 3.38$ (m, 7H, piperazine- $\left.\mathrm{CH}_{2}+\mathrm{NCH}_{3}\right) ;{ }^{13} \mathrm{C}$ NMR $(101 \mathrm{MHz}$, $\left.\mathrm{CDCl}_{3}\right) \delta: 159.2,156.1,155.0,151.8,147.9,147.4,137.7$, 114.1, 107.4, 105.5, 49.5, 44.9, 32.5, 29.7, 27.8; HRMS (Maldi) calcd for $\mathrm{C}_{17} \mathrm{H}_{22} \mathrm{~N}_{7} \mathrm{O}_{2}[\mathrm{M}+\mathrm{H}]^{+}$356.1829, found 356.1829 .

8-(4-(5-氯吡啶-2-基)哌嗪-1-基)-1,3,7-三甲基-3,7-二 氢- $1 H$-嘌呤-2,6-二酮(Ii): 白色固体, 产率 49.8\%. m.p. $184{ }^{\circ} \mathrm{C}$; ${ }^{1} \mathrm{H}$ NMR $\left(400 \mathrm{MHz}, \mathrm{CDCl}_{3}\right) \delta: 8.10(\mathrm{~s}, 1 \mathrm{H}$, pyridine-H), 7.43 (dd, $J=8.8,1.6 \mathrm{~Hz}, 1 \mathrm{H}$, Pyridine-H), $6.61(\mathrm{~d}, J=9.2 \mathrm{~Hz}, 1 \mathrm{H}$, pyridine- $\mathrm{H}), 3.77\left(\mathrm{~s}, 3 \mathrm{H}, \mathrm{NCH}_{3}\right)$, $3.66 \sim 3.63\left(\mathrm{~m}, 4 \mathrm{H}\right.$, piperazine- $\left.\mathrm{CH}_{2}\right), 3.49\left(\mathrm{~s}, 3 \mathrm{H}, \mathrm{NCH}_{3}\right)$, 3.35 (br, $7 \mathrm{H}$, piperazine- $\left.\mathrm{CH}_{2}+\mathrm{NCH}_{3}\right) ;{ }^{13} \mathrm{C}$ NMR $(101$ $\left.\mathrm{MHz}, \mathrm{CDCl}_{3}\right) \delta: 157.5,155.9,155.0,151.7,147.3,146.4$, 137.3, 120.9, 107.9, 105.5, 49.4, 44.9, 32.5, 29.7, 27.8; HRMS (Maldi) calcd for $\mathrm{C}_{17} \mathrm{H}_{20} \mathrm{ClN}_{7} \mathrm{NaO}_{2}[\mathrm{M}+\mathrm{Na}]^{+}$ 412.1259, found 412.1257.

1,3,7-三甲基-8-(4-(5-甲基嘧啶-2-基)哌嗪-1-基)-3,7二氢- $1 H$-嘌呤-2,6-二酮 $(\mathbf{I j})$ : 白色固体, 产率 $41.6 \%$. m.p. $245 \sim 247{ }^{\circ} \mathrm{C}$; ${ }^{1} \mathrm{H}$ NMR $\left(400 \mathrm{MHz}, \mathrm{CDCl}_{3}\right) \delta: 8.21(\mathrm{~s}, 2 \mathrm{H}$, pyrimidine-H), $3.99 \sim 3.93\left(\mathrm{~m}, 4 \mathrm{H}\right.$, piperazine- $\left.\mathrm{CH}_{2}\right), 3.82$ (s, $\left.3 \mathrm{H}, \mathrm{N}-\mathrm{CH}_{3}\right), 3.54$ (s, $\left.3 \mathrm{H}, \mathrm{NCH}_{3}\right), 3.42\left(\mathrm{~s}, 3 \mathrm{H}, \mathrm{NCH}_{3}\right)$, $3.37 \sim 3.32\left(\mathrm{~m}, 4 \mathrm{H}\right.$, piperazine- $\left.\mathrm{CH}_{2}\right), 2.17(\mathrm{~s}, 3 \mathrm{H}$, pyrimidine- $\left.\mathrm{CH}_{3}\right) ;{ }^{13} \mathrm{C}$ NMR $\left(101 \mathrm{MHz}, \mathrm{CDCl}_{3}\right) \delta: 157.8$, 156.2, 155.0, 151.8, 147.4, 119.0, 105.5, 49.6, 43.6, 32.5, 29.7, 27.8, 14.6; HRMS (Maldi) calcd for $\mathrm{C}_{17} \mathrm{H}_{22} \mathrm{~N}_{8} \mathrm{NaO}_{2}$ $[\mathrm{M}+\mathrm{Na}]^{+} 393.1758$, found 393.1753 .

8-(4-(5-甲氧基嘧啶-2-基)哌嗪-1-基)-1,3,7-三甲基3,7-二氢-1 $H$-嘌呤-2,6-二酮(Ik): 白色固体, 产率 $51.5 \%$. m.p. $265 \sim 267{ }^{\circ} \mathrm{C} ;{ }^{1} \mathrm{H}$ NMR $\left(400 \mathrm{MHz}, \mathrm{CDCl}_{3}\right) \delta: 8.14$ (s, $2 \mathrm{H}$, pyrimidine- $\mathrm{H}), 3.93 \sim 3.88\left(\mathrm{~m}, 4 \mathrm{H}\right.$, piperazine- $\left.\mathrm{CH}_{2}\right)$, $3.84\left(\mathrm{~s}, 3 \mathrm{H}, \mathrm{OCH}_{3}\right), 3.82\left(\mathrm{~s}, 3 \mathrm{H}, \mathrm{NCH}_{3}\right), 3.55(\mathrm{~s}, 3 \mathrm{H}$, 
$\left.\mathrm{NCH}_{3}\right), 3.41\left(\mathrm{~s}, 3 \mathrm{H}, \mathrm{NCH}_{3}\right), 3.37 \sim 3.32(\mathrm{~m}, 4 \mathrm{H}$, piperazine- $\left.\mathrm{CH}_{2}\right) ;{ }^{13} \mathrm{C}$ NMR $\left(101 \mathrm{MHz}, \mathrm{CDCl}_{3}\right) \delta: 157.7,156.2$, 155.0, 151.8, 147.4, 146.8, 144.8, 105.5, 56.9, 49.6, 44.1, 32.5, 29.7, 27.8; HRMS (Maldi) calcd for $\mathrm{C}_{17} \mathrm{H}_{22} \mathrm{~N}_{8} \mathrm{NaO}_{3}$ $[\mathrm{M}+\mathrm{Na}]^{+}$409.1707, found 409.1709.

1,3,7-三甲基-8-(4-(4-(三氟甲基)嘧啶-2-基)哌嗪-1基)-3,7-二氢- $1 H$-嘌呤-2,6-二酮(II): 白色固体, 产率 47.3\%. m.p. $210 \sim 211{ }^{\circ} \mathrm{C} ;{ }^{1} \mathrm{H}$ NMR (400 MHz, $\left.\mathrm{CDCl}_{3}\right) \delta$ : 8.54 (d, $J=4.8 \mathrm{~Hz}, 1 \mathrm{H}$, Pyrimidine-H), 6.83 (d, $J=4.8 \mathrm{~Hz}$, $1 \mathrm{H}$, Pyrimidine- $\mathrm{H}), 4.05(\mathrm{t}, J=5.2 \mathrm{~Hz}, 4 \mathrm{H}$, piperazine$\mathrm{CH}_{2}$ ), 3.82 (s, $3 \mathrm{H}, \mathrm{NCH}_{3}$ ), 3.53 (s, $3 \mathrm{H}, \mathrm{NCH}_{3}$ ), 3.40 (s, $3 \mathrm{H}$, $\left.\mathrm{NCH}_{3}\right), 3.34$ (t, $J=5.2 \mathrm{~Hz}, 4 \mathrm{H}$, piperazine- $\left.\mathrm{CH}_{2}\right) ;{ }^{13} \mathrm{C} \mathrm{NMR}$ $\left(101 \mathrm{MHz}, \mathrm{CDCl}_{3}\right) \delta: 161.5,160.2,156.2$ (q, $\left.J=35.4 \mathrm{~Hz}\right)$, 155.9, 154.9, 151.7, 147.3, 120.5 (q, $J=276.7 \mathrm{~Hz}), 105.5$, 105.2, 49.5, 43.2, 32.4, 29.7, 27.7; HRMS (Maldi) calcd for $\mathrm{C}_{17} \mathrm{H}_{19} \mathrm{~F}_{3} \mathrm{~N}_{8} \mathrm{NaO}_{2} \quad[\mathrm{M}+\mathrm{Na}]^{+}$447.1475, found 447.1473.

\subsection{6目标化合物 $\mathbf{I m} \sim \mathbf{I p}$ 的合成}

向 $25 \mathrm{~mL}$ 圆底烧瓶中加入中间体 $8(1 \mathrm{mmol})$ 、酰氯 (1 mmol) 和 $5 \mathrm{~mL}$ 二氯甲烷, 磁力搅拌使固体溶解, 再加 入三乙胺 $(3 \mathrm{mmol})$, 室温搅拌 $5 \mathrm{~h}$. 将体系倒入 $30 \mathrm{~mL}$ 水 中, 用二氯甲烷萃取 $(20 \mathrm{~mL} \times 3)$, 合并有机相, 用无水 硫酸钠干燥后减压脱溶, 残余物经柱色谱分离, 用二氯 甲烷/甲醇 $(V: V=30: 1)$ 洗脱, 得到目标化合物 $\mathbf{I m} \sim$ Ip.

8-(4-(4-甲氧基苯甲酰基)哌嗪-1-基)-1,3,7-三甲基3,7-二氢-1 $H$-嘌呤-2,6-二酮(Im): 白色固体, 产率 90.3\%. m.p. $210 \sim 213{ }^{\circ} \mathrm{C} ;{ }^{1} \mathrm{H}$ NMR $\left(400 \mathrm{MHz}, \mathrm{CDCl}_{3}\right) \delta$ : 7.42 (d, $J=8.7 \mathrm{~Hz}, 2 \mathrm{H}, \mathrm{PhH}), 6.94$ (d, $J=8.6 \mathrm{~Hz}, 2 \mathrm{H}, \mathrm{PhH}$ ), $3.85\left(\mathrm{~s}, 3 \mathrm{H}, \mathrm{OCH}_{3}\right), 3.82 \sim 3.66\left(\mathrm{~m}, 7 \mathrm{H}\right.$, piperazine- $\mathrm{CH}_{2}+$ $\left.\mathrm{NCH}_{3}\right), 3.52\left(\mathrm{~s}, 3 \mathrm{H}, \mathrm{NCH}_{3}\right), 3.39$ (s, $\left.3 \mathrm{H}, \mathrm{NCH}_{3}\right), 3.36 \sim$ 3.17 (m, 4H, piperazine- $\left.\mathrm{CH}_{2}\right) ;{ }^{13} \mathrm{C}$ NMR $(101 \mathrm{MHz}$, $\left.\mathrm{CDCl}_{3}\right) \delta: 170.6,161.0,155.5,155.0,147.2,129.2,127.1$, 113.8, 105.5, 55.4, 49.9, 32.3, 29.7, 27.8; HRMS (Maldi) calcd for $\mathrm{C}_{20} \mathrm{H}_{24} \mathrm{~N}_{6} \mathrm{NaO}_{4}[\mathrm{M}+\mathrm{Na}]^{+}$435.1751, found 435.1749 .

1,3,7-三甲基-8-(4-(4-硝基苯甲酰基)哌嗪-1-基)-3,7二氢- $1 H$-嘌呤-2,6-二酮(In): 黄色固体, 产率 $96.2 \%$. m.p. 206 207 ${ }^{\circ} \mathrm{C} ;{ }^{1} \mathrm{H}$ NMR (400 MHz, $\left.\mathrm{CDCl}_{3}\right) \delta: 8.30$ (d, $J=7.6 \mathrm{~Hz}, 2 \mathrm{H}, \mathrm{PhH}), 7.59$ (d, $J=7.7 \mathrm{~Hz}, 2 \mathrm{H}, \mathrm{PhH})$, 3.95 (br, $4 \mathrm{H}$, piperazine- $\left.\mathrm{CH}_{2}\right), 3.77\left(\mathrm{~s}, 3 \mathrm{H}, \mathrm{NCH}_{3}\right), 3.54$ (br, $2 \mathrm{H}$, piperazine- $\left.\mathrm{CH}_{2}\right), 3.49$ (s, $\left.3 \mathrm{H}, \mathrm{NCH}_{3}\right), 3.37$ (s, $3 \mathrm{H}$, $\mathrm{NCH}_{3}$ ), 3.28 (br, $J=31.6 \mathrm{~Hz}, 2 \mathrm{H}$, piperazine- $\mathrm{CH}_{2}$ ); ${ }^{13} \mathrm{C}$ NMR $\left(101 \mathrm{MHz}, \mathrm{CDCl}_{3}\right) \delta: 168.2,155.1,155.1,151.7$, 148.6, 147.1, 141.3, 128.2, 124.1, 105.6, 49.9, 32.3, 29.7,
27.8; HRMS (Maldi) calcd for $\mathrm{C}_{20} \mathrm{H}_{25} \mathrm{~N}_{6} \mathrm{O}_{4}[\mathrm{M}+\mathrm{H}]^{+}$ 428.1677, found 428.1677 .

8-(4-肉桂酰基哌嗪-1-基)-1,3,7-三甲基-3,7-二氢1H-嘌呤-2,6-二酮(Io): 白色固体, 产率 79.6\%. m.p. 212 213 ${ }^{\circ} \mathrm{C} ;{ }^{1} \mathrm{H}$ NMR $\left(400 \mathrm{MHz}, \mathrm{CDCl}_{3}\right) \delta: 7.72(\mathrm{~d}, J=$ $15.4 \mathrm{~Hz}, 1 \mathrm{H}, \mathrm{CH}=\mathrm{CH}), 7.59 \sim 7.49(\mathrm{~m}, 2 \mathrm{H}, \mathrm{PhH}), 7.47 \sim$ $7.33(\mathrm{~m}, 3 \mathrm{H}, \mathrm{PhH}), 6.89(\mathrm{~d}, J=15.4 \mathrm{~Hz}, 1 \mathrm{H}, \mathrm{CH}=\mathrm{CH})$, 3.87 (br, $4 \mathrm{H}$, piperazine- $\left.\mathrm{CH}_{2}\right), 3.80\left(\mathrm{~s}, 3 \mathrm{H}, \mathrm{NCH}_{3}\right), 3.53$ (s, $\left.3 \mathrm{H}, \mathrm{NCH}_{3}\right), 3.40\left(\mathrm{~s}, 3 \mathrm{H}, \mathrm{NCH}_{3}\right), 3.32$ (br, $4 \mathrm{H}$, piperazine$\left.\mathrm{CH}_{2}\right) ;{ }^{13} \mathrm{C}$ NMR (101 MHz, $\left.\mathrm{CDCl}_{3}\right) \delta: 165.7,155.4,155.0$, $151.7,143.6,134.9,129.9,128.8,127.8,116.4,105.6$, 99.9, 49.8, 32.4, 29.7, 27.8; HRMS (Maldi) calcd for $\mathrm{C}_{21} \mathrm{H}_{25} \mathrm{~N}_{6} \mathrm{O}_{3}[\mathrm{M}+\mathrm{H}]^{+}$409.1983, found 409.1983 .

8-(4-(3-澳-1-(3-氯吡啶-2-基)-1 $H$-吡唑-5-甲酰基)哌 嗪-1-基)-1,3,7-三甲基-3,7-二氢-1 $H$-嘌呤-2,6-二酮(Ip): 浅粉色固体, 产率 68.7\%. m.p. $229 \sim 230{ }^{\circ} \mathrm{C} ;{ }^{1} \mathrm{H}$ NMR $\left(400 \mathrm{MHz}, \mathrm{CDCl}_{3}\right) \delta: 8.46 \sim 8.39(\mathrm{~m}, 1 \mathrm{H}$, pyridine-H), 7.92 (d, $J=8.0 \mathrm{~Hz}, 1 \mathrm{H}$, pyridine-H), 7.37 (dd, $J=8.0,4.7$ $\mathrm{Hz}, 1 \mathrm{H}$, pyridine-H), 6.57 (s, 1H, pyrazole-H), 3.84 (br, $4 \mathrm{H}$, piperazine- $\left.\mathrm{CH}_{2}\right), 3.78\left(\mathrm{~s}, 3 \mathrm{H}, \mathrm{NCH}_{3}\right), 3.53(\mathrm{~s}, 3 \mathrm{H}$, $\mathrm{NCH}_{3}$ ), 3.39 (s, $3 \mathrm{H}, \mathrm{NCH}_{3}$ ), 3.31 (br, 4H, piperazine- $\mathrm{CH}_{2}$ ); ${ }^{13} \mathrm{C}$ NMR (101 MHz, $\left.\mathrm{CDCl}_{3}\right) \delta: 159.5,155.2,155.0,151.7$, $147.6,147.1,146.5,140.1,138.8,127.8,127.2125 .2$, 110.2, 105.6, 49.6, 32.35, 29.7, 27.8; HRMS (Maldi) calcd for $\mathrm{C}_{21} \mathrm{H}_{22} \mathrm{BrClN}_{9} \mathrm{O}_{3}[\mathrm{M}+\mathrm{H}]^{+}$562.0712, found562.0718.

3.2.7目标化合物 Ia Ip 的生物活性测试

杀虫活性参照文献方法 ${ }^{[23-24]}$ 对方法对 3 4 龄东方 粘虫 (Mythimna separata Walker) 和小菜蛾 (Plutella xylostella L.)进行测试. 抑菌测试采用离体平皿法 ${ }^{[25]}$, 在 $50 \mathrm{mg} \cdot \mathrm{L}^{-1}$ 浓度下, 分别对黄瓜灰霉病菌(Botrytis cinerea)、油菜菌核病菌(Sclerotinia sclerotiorum)、苹果 轮纹病菌 (Physalospora piricola)、番茄早疫病菌 (Alternaria solani Sorauer)、小麦纹枯病菌(Rhizoctonia cerealis)和辣椒疫霉病菌(Phytophthora capsici) 6 种常见 病菌进行了测试.

辅助材料(Supporting Information) 中间体 7、8 的 ${ }^{1} \mathrm{H}$ NMR、 ${ }^{13}$ C NMR 谱图, 目标化合物 $\mathbf{I a} \sim \mathbf{I p}$ 的 ${ }^{1} \mathrm{H}$ NMR、 ${ }^{13} \mathrm{C}$ NMR 谱图，以及化合物 Ip 的晶体数据. 这些材料可 以免费从本刊网站(http://sioc-journal.cn/)上下载.

\section{References}

[1] Oerke, E. C.; Dehne, H. W. Crop Prot. 2004, 23, 275.

[2] Liu, X.-H.; Fang, Y.-M.; Xie, F.; Zhang, R.-R.; Shen, Z.-H.; Tan, C.-X.; Weng, J.-Q.; Xu, T.-M.; Huang, H.-Y. Pest Manage. Sci. 2017, 73, 1900.

[3] Singh, N.; Shreshtha, A. K.; Thakur, M. S.; Patra, S. Heliyon 2018 , 
4, e00829.

[4] Hasegawa, T.; Nadai, M.; Apichartpichean, R.; Muraoka, I.; Nabeshima, T.; Takagi, K. J. Pharm. Sci. 1991, 80, 962.

[5] Mielke, R.; Kittner, B.; Ghaemi, M.; Kessler, J.; Szelies, B.; Herholz, K.; Heiss, W. D. J. Neurol. Sci. 1996, 141, 59.

[6] Rieg, T.; Steigele, H.; Schnermann, J.; Richter, K.; Osswald, H.; Vallon, V. J. Pharmacol. Exp. Ther. 2005, 313, 403.

[7] Yeh, J.-L.; Liu, C.-P.; Hsu, J.-H.; Tseng, C.-J.; Wu, P.-J.; Wang, Y.-Y.; Wu, J.-R.; Chen, I.-J. Kaohsiung J. Med. Sci. 2012, 28, 567.

[8] Gressel, J. Pest Manage. Sci. 2018, 74, 511.

[9] Nathanson, J. A. Science 1984, 226, 184.

[10] Schmitt, M.; Turberg, A.; Londershausen, M.; Dorn, A. Pestic. Sci. 1996, $48,375$.

[11] Suzuki, H.; Utsunomiya, I.; Shudo, K.; Fukuhara, N.; Iwaki, T.; Yasukata, T. Eur. J. Med. Chem. 2013, 69, 262.

[12] Nagesh, H. N.; Suresh, A.; Sairam, S. D. S. S.; Sriram, D.; Yogeeswari, P.; Chandra Sekhar, K. V. G. Eur. J. Med. Chem. 2014, 84, 605.

[13] Bourke, J. B.; Nelsen, T. R.; Eichler, D. J. Agric. Food Chem. 1977, $25,36$.

[14] Wang, B.-L.; Liu, X.-H.; Zhang, X.-L.; Zhang, J.-F.; Song, H.-B.; Li, Z.-M. Chem. Biol. Drug Des. 2011, 78, 42.

[15] Wang, B.-L.; Shi, Y.-X.; Zhang, S.-J.; Ma, Y.; Wang, H.-X.; Zhang, L.-Y.; Wei, W.; Liu, X.-H.; Li, Y.-H.; Li, Z.-M.; Li, B.-J. Eur. J. Med. Chem. 2016, 117, 167.

[16] Zhang, Y.; Liu, X.-H.; Zhan, Y.-Z.; Zhang, L.-Y.; Li, Z.-M.; Li,
Y.-H.; Zhang, X.; Wang, B.-L. Bioorg. Med. Chem. Lett. 2016, 26, 4661.

[17] Zhang, Y.; Zhan, Y.-Z.; Ma, Y.; Hua, X.-W.; Wei, W.; Zhang, X.; Song, H.-B.; Li, Z.-M.; Wang, B.-L. Chin. Chem. Lett. 2018, 29, 441.

[18] Wang, B.-L.; Shi, Y.-X.; Ma, Y.; Liu, X.-H.; Li, Y.-H.; Song, H.-B.; Li, B.-J.; Li, Z.-M. J. Agric. Food Chem. 2010, 58, 5515.

[19] Zhang, Y.; Li, Z.; Song, H.; Wang, B. Chin. J. Chem. 2018, 36, 635.

[20] Li, G.; Meng, B.; Yuan, B.; Huan, Y.; Zhou, T.; Jiang, Q.; Lei, L.; Sheng, L.; Wang, W.; Gong, N.; Lu, Y.; Ma, C.; Li, Y.; Shen, Z.; Huang, H. Eur. J. Med. Chem. 2020, 188, 112017.

[21] Liu, H.; Zhang, S.; Li, H.; Zhang, Y.; Li, Z.; Wang, B. Chin. J. Org. Chem. 2021, 41, 2091 (in Chinese) (刘航, 张舒昀, 李欢, 张燕, 李正名, 王宝雷, 有机化学, 2021, 41, 2091.)

[22] Zimmer, H.; Amer, A.; Baumann, F. M.; Haecker, M.; Hess, C. G. M.; Ho, D.; Huber, H. J.; Koch, K.; Mahnke, K.; Schumacher, C.; Wingfield, R. C. Eur. J. Org. Chem. 1999, 9, 2419.

[23] Wang, B.-L.; Zhu, H.-W.; Ma, Y.; Xiong, L.-X.; Li, Y.-Q.; Zhao, Y.; Zhang, J.-F.; Chen, Y.-W.; Zhou, S.; Li, Z.-M. J. Agric. Food Chem. 2013, 61, 5483.

[24] Wang, B.; Wang, H.; Liu, H.; Xiong, L.; Yang, N.; Zhang, Y.; Li, Z. Chin. Chem. Lett. 2020, 31, 739.

[25] Wang, B.-L.; Zhu, H.-W.; Li, Z.-M.; Wang, L.-Z.; Zhang, X.; Xiong, L.-X.; Song, H.-B. Pest Manage. Sci. 2018, 74, 726. 